\title{
miR-146a-3p suppressed the differentiation of hAMSCs into Schwann cells via inhibiting the expression of ERBB2
}

\author{
Wei Chen ${ }^{1} \cdot$ Linlin $^{\mathrm{Ji}^{1}} \cdot$ Zairong Wei $^{1}$ (D) Chenglan Yang ${ }^{1}$. Shusen Chang ${ }^{1} \cdot$ Yucheng Zhang $^{1} \cdot \mathrm{Kaiyu} \mathrm{Nie}^{1}$. \\ Lingli Jiang ${ }^{1} \cdot$ Yurong Deng ${ }^{1}$
}

Received: 5 December 2019 / Accepted: 12 October 2020 / Published online: 15 January 2021

(c) The Author(s) 2021

\begin{abstract}
Human amniotic mesenchymal stem cells (hAMSCs) can be differentiated into Schwann-cell-like cells (SCLCs) in vitro. However, the underlying mechanism of cell differentiation remains unclear. In this study, we explored the phenotype and multipotency of hAMSCs, which were differentiated into SCLCs, and the expression of nerve repair-related Schwann markers, such as S100 calcium binding protein B (S-100), TNF receptor superfamily member 1B (P75), and glial fibrillary acidic protein (GFAP) were observed to be significantly increased. The secreted functional neurotrophic factors, like brain-derived neurotrophic factor (BDNF), nerve growth factor (NGF), and neurotrophin-3 (NT-3), were determined and also increased with the differentiation time. Moreover, miR-146a-3p, which significantly decreased during the differentiation of hAMSCs into SCLCs, was selected by miRNA-sequence analysis. Further molecular mechanism studies showed that Erb-B2 receptor tyrosine kinase 2 (ERBB2) was an effective target of miR-146a-3p and that miR-146a-3p down-regulated ERBB2 expression by binding to the 3'-UTR of ERBB2. The expression of miR-146a-3p markedly decreased, while the mRNA levels of ERBB2 increased with the differentiation time. The results showed that down-regulating miR-146a-3p could promote SC lineage differentiation and suggested that miR-146a-3p negatively regulated the Schwann-like phenotype differentiation of hAMSCs by targeting ERBB2. The results will be helpful to establish a deeper understanding of the underlying mechanisms and find novel strategies for cell therapy.
\end{abstract}

Keywords Human amniotic mesenchymal stem cells $\cdot$ Schwann cells $\cdot$ miR-146a-3p $\cdot$ ERBB2 $\cdot$ Neurotrophic factors

$\begin{array}{ll}\text { Abbreviations } \\ \text { hAMSCs } & \text { Human amniotic mesenchymal stem cells } \\ \text { BDNF } & \text { Brain-derived neurotrophic factor } \\ \text { GDNF } & \text { Glial cell-derived neurotrophic factor } \\ \text { NGF } & \text { Nerve growth factor } \\ \text { NT-3 } & \text { Neurotrophin-3 } \\ \text { DAPI } & \text { 4',6-Diamidino-2-phenylindole } \\ \text { ARS } & \text { Alizarin red S } \\ \text { ERBB2 } & \text { Erb-B2 receptor tyrosine kinase 2 } \\ \text { SCLCs } & \text { Schwann-cell-like cells } \\ \text { S100 } & \text { S100 calcium binding protein B } \\ \text { P75 } & \text { TNF receptor superfamily member 1B } \\ \text { KEGG } & \text { Kyoto Encyclopedia of Genes and Genomes } \\ \text { GO } & \text { Gene Ontology }\end{array}$

Zairong Wei

zairongwei@sina.com

1 Department of Burns and Plastic Surgery, Affiliated Hospital of Zunyi Medical College, 149 Dalian Road, Guizhou, Zunyi, China

\section{Introduction}

Schwann cells (SCs) play a key role in successful nerve repair and regeneration by supporting both axonal growth and myelination. The functions of SCs are myelin-sheath formation, nerve impulse transmission, and the secretion of a variety of neurotrophic factors, such as brain-derived neurotrophic factor (BDNF), glial cell-derived neurotrophic factor (GDNF), nerve growth factor (NGF), and neurotrophin-3 (NT-3), and of extracellular matrix components that provide a beneficial microenvironment for neuronal survival and axonal growth (Carr and Johnston 2017). However, the acquisition of human Schwann cell sources encounters some difficulties, such as their slow proliferation rate and limited in vitro culture. In clinical operations, autologous acquisition of SCs can cause peripheral nerve injury, but, at the same time, it also faces the problem of the lack of donors (Armaiz and Wang 2017; Jiang et al. 2017).

Adult stem cells, such as human amniotic mesenchymal stem cells (hAMSCs), which are derived from the amniotic 
membrane and amniotic fluid, have a great capacity for proliferation and differentiation and do not form teratomas when transplanted into animal models (Carbone et al. 2014; Diaz-Prado et al. 2011; Zheng et al. 2008). Recently, it has reported that Schwann cell-like cells (SCLCs) can be differentiated from hAMSCs (Jiang et al. 2010). However, the mechanism of differentiation has not been fully elucidated. Thus, the understanding of the molecular mechanism of the differentiation of SCLCs from hAMSCs might help to create more desirable procedures to obtain SCLC-derived lineages in vitro.

MicroRNAs (miRNAs) are a subset of RNAs that are short, non-coding RNAs of approximately 25 nucleotides, which have been shown to play multiple roles in the regulation of development and differentiation. Increasing evidences have confirmed that miRNAs participate in the differentiation of all three germ layers, including adipogenesis, osteogenesis, and myogenesis (Marti et al. 2016; Mok et al. 2017). Several miRNAs were demonstrated to be involved in the modulation of hAMSC differentiation: miR-125b positively regulated adipogenic differentiation by targeting PTEN, cyclin E1, and CDK6; miR-204 inhibited osteoblast differentiation from hAMSCs; miR-138/miR-222 over-expression contributed to hAMSC differentiation into adipocytes in pregnancy (Avendaño-Féli et al. 2019; Glemžaitė and Navakauskienė 2016; Nardelli et al. 2017; Trohatou et al. 2014, 2017). Nevertheless, seldom comprehensive analysis has been performed on the miRNAs involved in the differentiation of hAMSCs into SCLCs. Therefore, the miRNAs playing a role in the regulation and the mechanisms by which miRNAs regulate Schwann cell differentiation need to be further studied.

In this study, hAMSCs were cultured and differentiated into SCLCs, and the expression of the nerve repair-related Schwann markers S100 calcium-binding protein B (S-100), TNF receptor superfamily member $1 \mathrm{~B}(\mathrm{P} 75)$, and glial fibrillary acidic protein (GFAP) were observed to be significantly increased. The secreted functional neurotrophic factors BDNF, NGF, and NT3 were determined and were also observed to increase with the differentiation time. Then, the expression of miRNAs was profiled during hAMSC differentiation into Schwann cell-like cells, and miR-146a-3p was significantly decreased, which might indicate that it played a negative regulatory role in SCLC lineage differentiation. Furthermore, Erb-B2 receptor tyrosine kinase 2 (ERBB2), an important regulator of the SC lineage differentiated from hAMSCs, was identified as a direct target of miR-146a-3p. The results showed that the down-regulation of miR-146a-3p would promote SC lineage differentiation and suggested that miR-146a-3p negatively regulated the Schwannlike phenotype differentiation of hAMSCs by targeting ERBB2. The results will be helpful to establish a deeper understanding of the underlying mechanisms and find novel strategies for cell therapy.

\section{Materials and methods}

\section{Culture and differentiation of hAMSCs}

hAMSCs were purchased from SALIAI Stem Cell Technology (Guangzhou, China). The cells were cultured in LG-DMEM (Gibco, Grand Island, USA) medium (containing 10\% FBS, $2 \mathrm{mM}$ glutamine, $1 \%$ non-essential amino acids, $55 \mathrm{mM}$ 2-mercaptoethanol, $1 \mathrm{mM}$ sodium pyruvate, $100 \mathrm{U} / \mathrm{mL}$ penicillin, and $100 \mathrm{mg} / \mathrm{mL}$ streptomycin) and inoculated in 6-well culture plates at a cell density of $5 \times 10^{5} / \mathrm{mL}$, at $37^{\circ} \mathrm{C}$ with $5 \% \mathrm{CO}_{2}$. After the cell confluence reached $80-90 \%$, the cells were digested with $0.25 \%$ trypsin- $0.02 \%$ EDTA solution, at $37{ }^{\circ} \mathrm{C}$ for $2-3 \mathrm{~min}$. The effect of trypsin was terminated by adding culture medium. After centrifugation for $5 \mathrm{~min}$, the supernatant was discarded, and the cell pellet was resuspended in culture medium, the cells were subcultured at a cell density of $1 \times 10^{7} / \mathrm{mL}$. hAMSCs at passage 3 (P3) were used in the differentiation experiments. MiR146a-3p mimic and its negative control, and anti-miR-146a-3p inhibitor, ERBB2 siRNA and control siRNA were purchased from RiboBio (Guangzhou, China) and transfected with ribo FECT $^{\text {TM }}$ CP Transfection Kit (RiboBio, Guangzhou, China). All experiments and procedures were approved by the Ethics Committee of the Zunyi Medical University.

\section{Multipotential differentiation}

For adipogenic differentiation, hAMSCs were cultured in fat-inducing medium (Iscove's modified Dulbecco's media (IMDM) supplemented with $10 \% \mathrm{FBS}, 10 \mu \mathrm{g} / \mathrm{mL}$ insulin, $1 \mu \mathrm{M}$ dexamethasone, $0.5 \mathrm{mM} \mathrm{IBMX}$, and $0.1 \mathrm{mM}$ indomethacin) for 12 days. After lipid droplets were formed, oil red $\mathrm{O}$ staining was performed. Cells were washed three times with PBS and fixed for $20 \mathrm{~min}$ in $10 \%$ neutral formaldehyde. After washing twice with PBS, oil red O buffer (Millipore, Burlington, USA) was added for $30 \mathrm{~min}$, after which cells were washed again twice. Cells were, then, subjected to hematoxylin staining for $1 \mathrm{~min}$. The decontamination solution was discarded, and the cells were photographed using an inverted microscope.

For osteogenic differentiation, hAMSCs were cultured with osteogenic induction medium (IMDM supplemented with $10 \% \mathrm{FBS}, 5 \mu \mathrm{g} / \mathrm{mL}$ insulin, $0.1 \mu \mathrm{M}$ dexamethasone, $0.2 \mathrm{mM}$ vitamin $\mathrm{C}$, and $10 \mathrm{mM}$ beta-glycerophosphate) for 15 days. Alizarin red S (ARS) staining was performed after the formation of mineralized nodules. Cells were washed three times with PBS, fixed for $20 \mathrm{~min}$ in $10 \%$ neutral formaldehyde, and washed again twice with PBS. Cells were submerged in alizarin red S staining solution (Millipore, Burlington, USA) and exposed to sunlight for $30 \mathrm{~min}$. After washing twice with PBS, mineralized nodules were observed and photographed using an inverted microscope. 
For SCLC differentiation, hAMSCs were seeded into 6-well plates in regular culture medium. On the following day, cells were changed to differentiation basic medium DMEM/F12 contained $1 \mathrm{mmol} / \mathrm{L} \beta$-mercaptoethanol on the first day. After $24 \mathrm{~h}$, the medium was changed into DMEM/ F12 with $35 \mathrm{ng} / \mathrm{mL}$ ATRA and 10\% FBS for 3 days. After that, the medium was discarded, the cells were washed three times with PBS and changed with medium DMEM/ F12 with $5 \mathrm{ng} / \mathrm{ml}$ PDGF-AA (Peprotech, USA), $10 \mathrm{ng} / \mathrm{ml}$ bFGF (Peprotech, USA), 14 lM forskolin (Sigma, USA), and $200 \mathrm{ng} / \mathrm{ml}$ heregulin (Peprotech, USA). Thereafter, the medium was changed twice a week. Cells were cleaned twice with PBS and photographed under inverted microscope at day 0 , day 7 , day 14 , and day 21 .

\section{MiRNA-seq analysis}

To identify SCLC-related miRNAs, hAMSCs were exposed to SCLC induction medium, as described above, and total RNA was isolated using the TRIzol reagent (Invitrogen, Thermo Fisher Scientific, USA) at day 14. Sample processing, hybridization, and microRNA sequencing analysis were conducted by Majorbio Biotechnology (Shanghai, China). Briefly, a fold change $\geq 2$ or $\leq 0.5$ between hAMSC and induced Schwann cells samples and a $p$ value $<0.05$ were chosen as the cutoff criteria for differentially expressed miRNAs. Down-stream targets of miRNAs were predicted by Targetscan 7.2 (https://www.targetscan.org/ vert72/), miRTarbase (https://mirtarbase.mbc.nctu.edu. tw/), and DIANA TOOLS (https://diana.imis.athena-innov ation.gr/DianaTools/). Overlapping predicted targets of the top 10 differentially expressed miRNAs in different times were used in pathway enrichment analyses performed by using Metascape (https://www.metascape.org/).

\section{Flow cytometry}

Flow cytometry was used to identify the purity of P3 hAMSCs. Cultured cells were incubated with a primary antibody against CD44, CD90, CD73, or CD105 (Abcam, Boston, USA), for $1 \mathrm{~h}$ on ice, to confirm the expression of the cell surface biomarkers. After washing with PBS, cells were incubated with the fluorescent dye-conjugated Alexa-488 secondary antibody (Bio-Rad, Hercules, USA), for $30 \mathrm{~min}$ at $4{ }^{\circ} \mathrm{C}$. After that, cells were washed and stained with RNase and propidium iodide (PI), for $30 \mathrm{~min}$, to calculate the cell proportion in each cell cycle phase. Cells were analyzed using a FACSCalibur flow cytometer with the Cell Quest acquisition software (BD Biosciences, Franklin Lakes, USA). List mode files were analyzed with the FlowJo software (Tree Star, San Carlos, USA).

\section{Immunofluorescence}

To study the morphological changes of hAMSCs after differentiation, cells were washed three times with PBS and fixed in $4 \%$ cold paraformaldehyde for $15 \mathrm{~min}$. Cells were, then, incubated in $0.2 \%$ Triton X-100, for $10 \mathrm{~min}$, and blocked with $10 \%$ goat serum, for $60 \mathrm{~min}$ at $37^{\circ} \mathrm{C}$. Cells were incubated overnight at $4{ }^{\circ} \mathrm{C}$ with the following primary antibodies (all from Abcam, Boston, USA): rabbit anti-GFAP (1:1500), rabbit anti-P75 (1:500), and mouse anti-S100 (1:300). Cells were, then, incubated with Alexa Fluor 594- or Alexa Fluor 488-conjugated anti-mouse or anti-rabbit (1:300, Invitrogen, Carlsbad, USA) secondary antibodies. Finally, the cells were stained with DAPI/PI for $5 \mathrm{~min}$. All images were photographed using a fluorescence microscope.

\section{Quantitative real-time quantitative PCR}

Total RNA was extracted with TRIzol reagent and converted into cDNA using M-MLV cDNA kit (Invitrogen, Carlsbad, USA). The Homo sapiens (has)-miR-146a-3p RNA mimic and inhibitor, ERBB2 siRNAs, and control siRNAs were reversetranscribed using a specific RT primer (RiboBio, Guangzhou, China), according to the manufacturer's protocol. GAPDH was used as an endogenous normalization control for mRNAs, and U6 was used as endogenous normalization control for miR-146a-3p. Primer pairs for all mRNAs and miRNAs were designed using the RiboBio primer online design tool (RiboBio, Guangzhou, China). RT-qPCR was performed using the SYBR Green PCR Kit (Toyobo, Osaka, Japan) and the Applied Biosystems 7500 Real-Time PCR Detection System (Life Technologies, Carlsbad, USA). The data were analyzed using the $2^{-\Delta \Delta \mathrm{Ct}}$ relative expression method. All experiments were repeated three times. Primer sequences are shown in Table 1.

\section{Western blot analysis}

Total proteins were extracted from cells and tissues using RIPA solution (Beyotime, Shanghai, China), according to the manufacturer's instructions. Equivalent amounts of proteins from each sample were separated by $10 \%$ SDS-PAGE, transferred to $0.22 \mu \mathrm{m}$ PVDF membranes (Millipore, Burlington, USA), blocked in 5\% fat-free milk for $1 \mathrm{~h}$, and incubated with specific primary antibodies (all from Abcam, Boston, USA) as listed: GFAP (mouse monoclonal, 1:500), S100 (rabbit monoclonal, 1:1,000), p75NTR (rabbit polyclonal, 1:500). After the incubation with the primary antibodies, the membranes were incubated with HRP-conjugated IgG for $2 \mathrm{~h}$, followed by detection with an enhanced chemiluminescence system. A GAPDH antibody (1:500) was used as control. The experiment was performed in triplicate. 
Table 1 Primer sequence for RT-qPCR

\begin{tabular}{lll}
\hline Target gene & Direction & Sequence $\left(5^{\prime}-3^{\prime}\right)$ \\
\hline S-100 & Forward & GGAAATCAAAGAGCAGGAGGT \\
& Reverse & ATTAGCTACAACACGGCTGGA \\
GFAP & Forward & CCTCTCCCTGGCTCGAATG \\
& Reverse & GGAAGCGAACCTTCTCGATGTA \\
P75 & Forward & GGAAGCGAACCTTCTCGATGTA \\
& Reverse & TGAAGGCTATGTAGGCCACAA \\
ERBB2 & Forward & GACAACTAGTACCAGAAGGCCAAGTCCGCA \\
& Reverse & GACAAAGCTTAGCTGTTTTCCAAAATATAT \\
GAPDH & Forward & TCAAGAAGGTGGTGAAGCAG \\
& Reverse & CGTCAAAGGTGGAGGAGTG \\
miR-146a-3p & Forward & CAGCCTCTGAAATTCAGTTCT \\
& Reverse & TCCAGTTTTTTTTTTTTTCTGAAGA \\
\hline
\end{tabular}

$R T$ - $q P C R$ reverse transcription quantitative polymerase chain reaction, $S$ - $100 \mathrm{~S} 100$ calcium-binding protein $\mathrm{B}, P 75$ TNF receptor superfamily member 1B, GFAP glial fibrillary acidic protein, ERBB2 Erb-B2 receptor tyrosine kinase 2, GAPDH glyceraldehyde-3-phosphate dehydrogenase

\section{ELISA assay}

The supernatants of the third generation of hAMSCs and neuron-like cells at days 3, 7, 14, and 21 after differentiation were collected, and the levels of BDNF, NGF, and NT3 in the medium from different groups were quantified using ELISA kits (Cusabio Technology, Wuhan, China), according to the manufacturer's instructions.

\section{Statistical analysis}

All data were expressed as the mean \pm standard deviation (SD) of the mean. Data were analyzed by one-way analysis of variance (ANOVA). In comparisons involving $\geq \mathrm{d} 3$ groups, one-way ANOVA followed by Bonferroni Dunn tests was used. A $p$ value $<0.05$ was considered statistically significant in two-tailed ANOVA.

\section{Results}

\section{Characterization of hAMSCs and detection of osteogenic and adipogenic abilities of hAMSC}

HAMSCs were cultured in Schwann cells induction medium in vitro. After $48 \mathrm{~h}$ of hAMSC culture, the cells grew mostly oval and triangular in shape. After 6 days in culture, the cells could proliferate and be passaged successfully. After passaging, cells, especially the third-generation ones (P3), grew rapidly with high purity and aligned tightly, presenting a fibroblast-like shape as previous paper described (Romani et al. 2015; Wang et al. 2020). The sixth-generation (P6) cells grew in a spiral colony, and their morphology changed clearly to a flat shape (Fig. 1a). Therefore, P3 hAMSCs were used for further research.

CD44, CD73, CD90, and CD105 are specific markers of hAMSCs (Dominici et al. 2006; Koik et al. 2014). Therefore, hAMSCs were clearly identified by detecting the expression of the mentioned markers through flow cytometry. Differentiated cells that did not express CD44, CD73, CD90, and CD105 were used as negative controls. Results showed that CD44, CD73, CD90, and CD105 were all highly expressed (> 90\%) in cultured hAMSCs (Fig. 1c-f). After 21 days of in vitro induction, the differentiation capability of the hAMSCs was detected with Alizarin red S and oil red O. hAMSCs grew from long spindle shapes into polygons or triangles in morphology, and Alizarin red S staining was positive, showing obvious osteogenic differentiation. After in vitro induction with adipogenic medium, red lipid droplets could be seen after oil red $\mathrm{O}$ staining in induced hAMSCs (Fig. 1b). The above results suggested that hAMSCs had good stemness and differentiation potential.

\section{The expression of S-100, P75, and GFAP were significantly increased after differentiation into SCLCs, and BDNF, NGF and NT3 expressions also markedly increased during differentiation}

The hAMSCs with high purity and uniform shapes were used for Schwann-like cell differentiation. After 7 days of differentiation, the cells adhered to the wall and grew mostly in a strip shape. After 14 days of differentiation, the strip shape of cells was more obvious, and their growth rate was slower. At day 21 after differentiation, the cells were single, flat, and slow growing (Fig. 2a). 

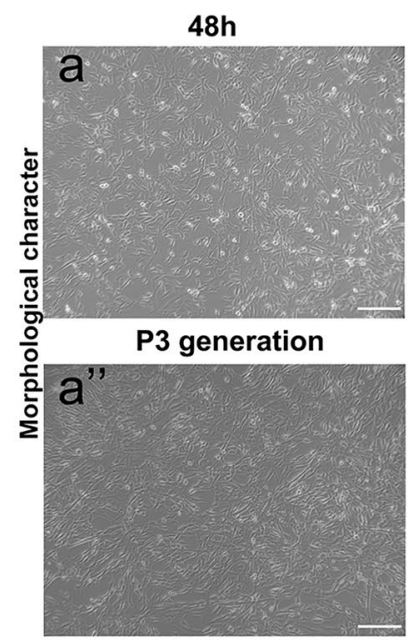

NC

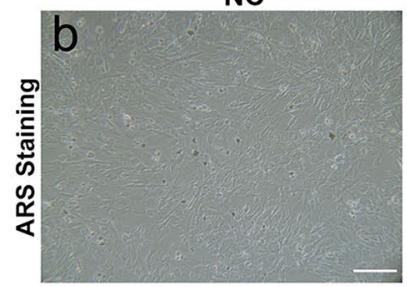

NC

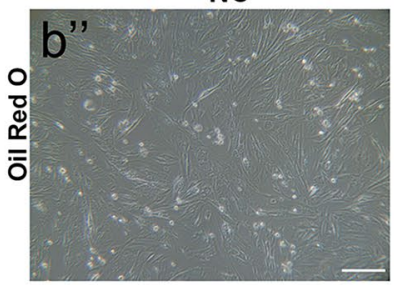

6 days

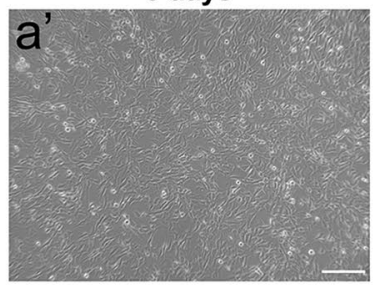

P6 generation

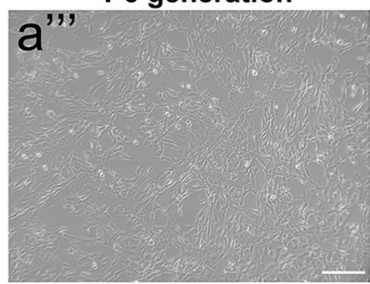

Osteogenic induction

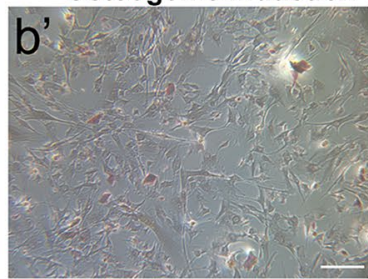

Adipogenesis induction

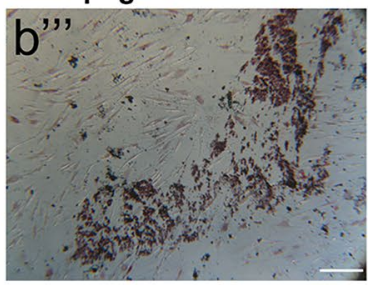

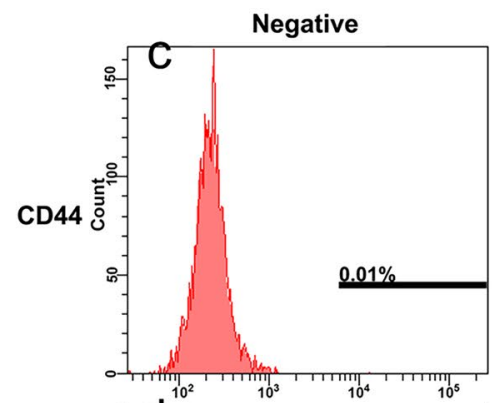
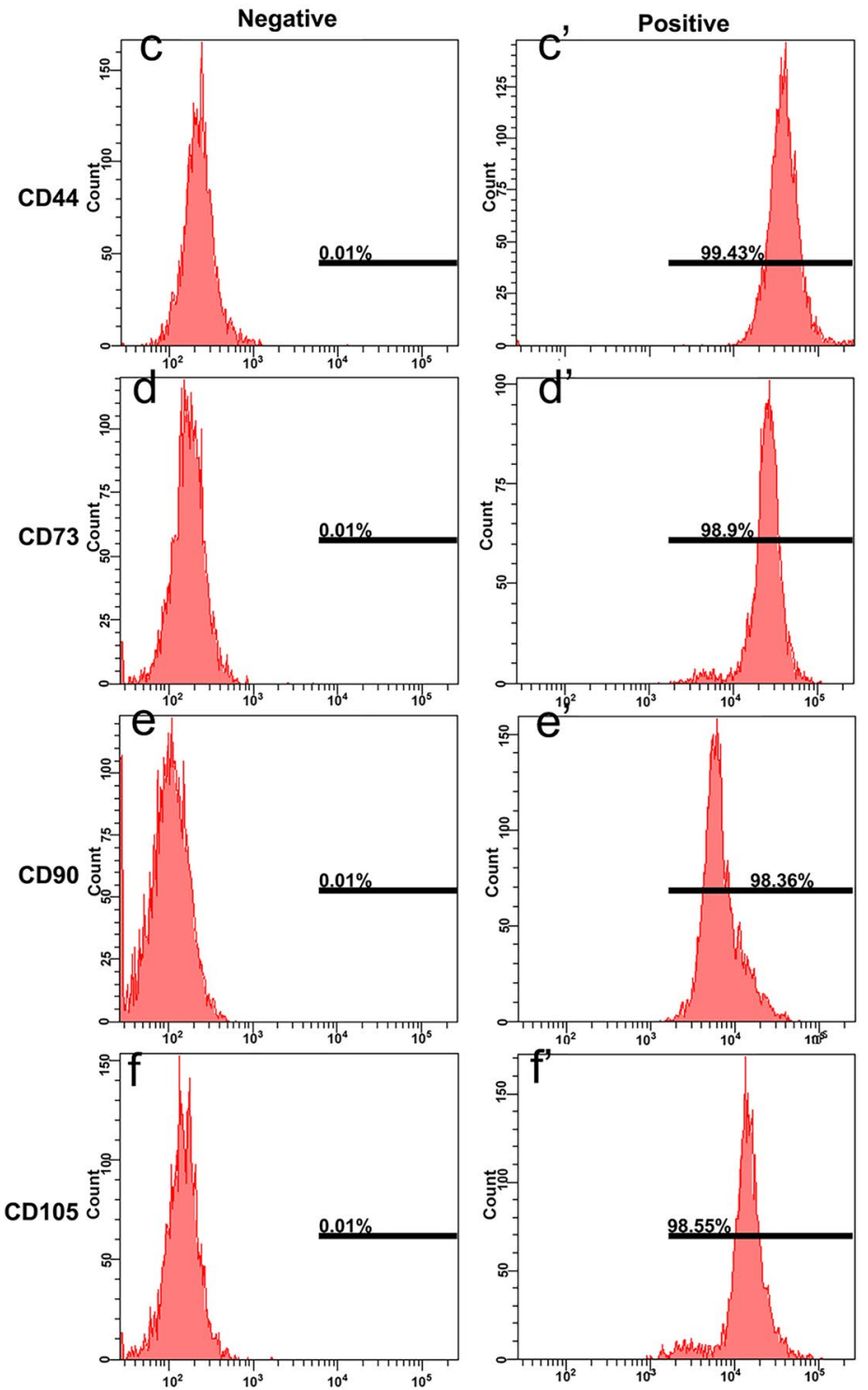

Fig. 1 Characterization of hAMSCs and detection of osteogenic and adipogenic abilities of hAMSCs. (a) The culture and morphological identification of hAMSCs (scale bar $100 \mu \mathrm{m}$ ). The morphological characters of hAMSCs of $48 \mathrm{~h}$, day 6, P3 generation and P6 generation. (b) Alizarin red S (ARS) and oil red $\mathrm{O}$ staining were used

In order to determine whether the hAMSCs had the differentiation potential to neuronal-like cells, the presence of the neurocyte-specific proteins S-100, P75, and GFAP was assessed in SCLCs 21 days after induction by immunofluorescence. The results showed that S-100, P75, and GFAP were more abundantly expressed in SCLCs after induction than in hAMSCs before induction (Fig. 2b-d). The mRNA and protein levels of S-100, P75, and GFAP were to detect the osteogenic and adipogenic differentiation of hAMSCs (scale bar $100 \mu \mathrm{m})$. (c-f) The expression of hAMSCs marker genes in P3 generation hAMSCs investigated by flow cytometry. CD44 (c), CD73 (d), CD90 (e), and CD105 (f) were all positive

also investigated to further confirm the above results. Results from qPCR and western bolt showed that the expressions of S-100, P75, and GFAP in cells were significantly increased after differentiation $(p<0.05)$ (Fig. 2 e and f). These results indicated that hAMSCs could be differentiated into iSCs successfully.

During the differentiation from hAMSCs into SCLCs, the supernatants were collected, and the levels of BDNF, 

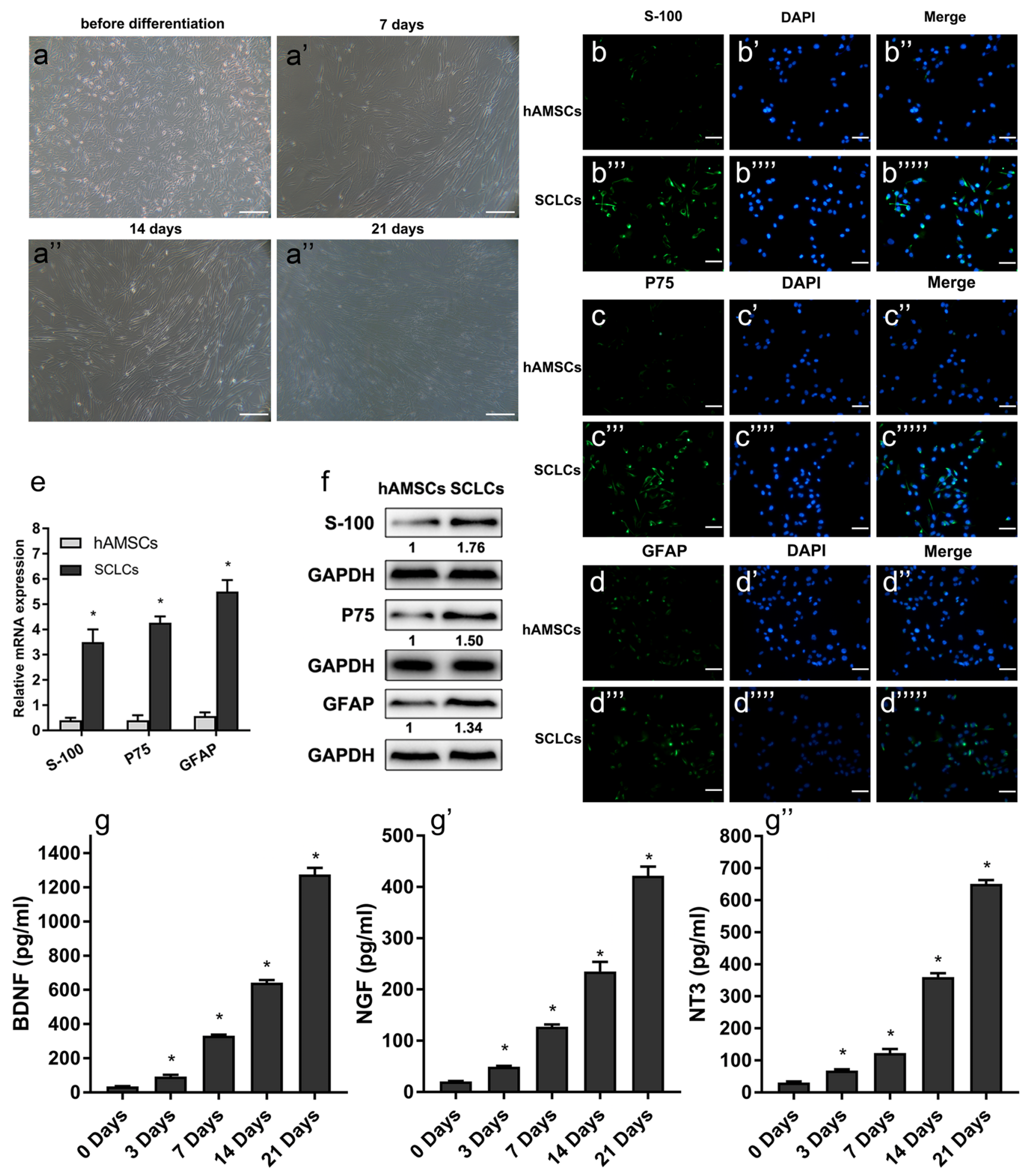

DAPI
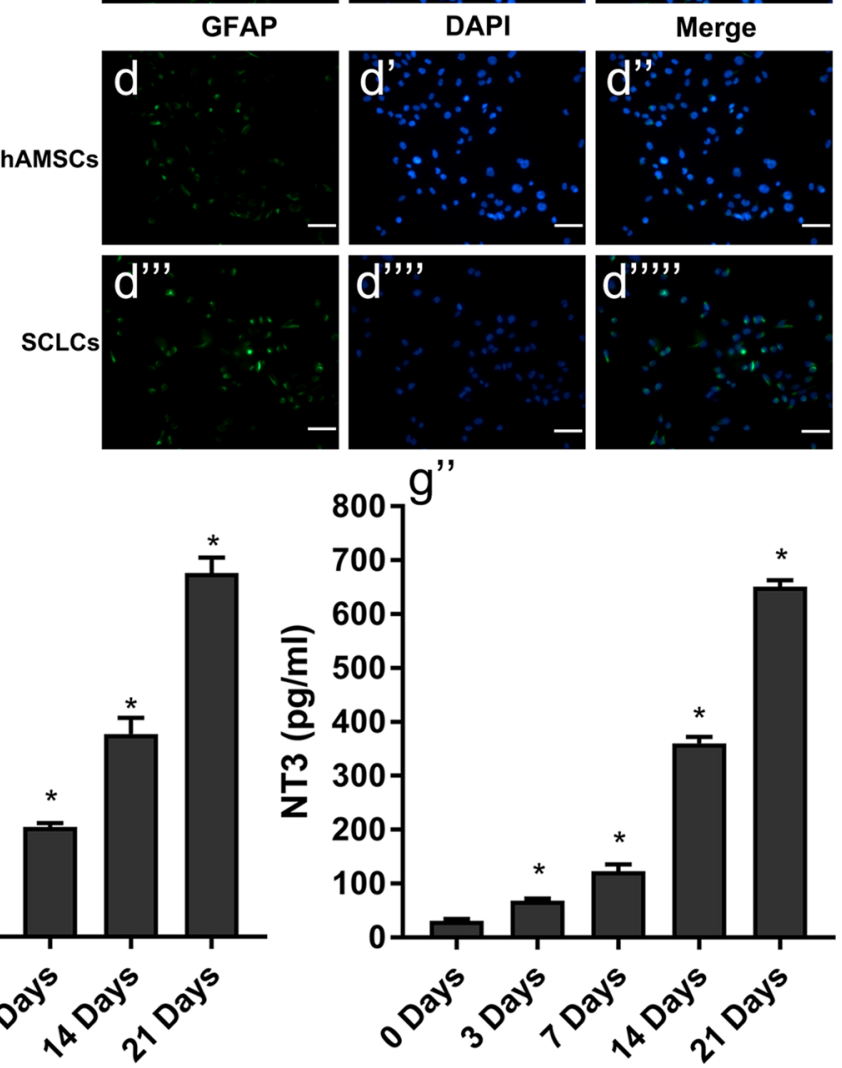

NGF, and NT3 in the supernatants were determined. The levels of BDNF, NGF, and NT3 in the supernatant increased significantly at day 3 and increased with the prolongation of the induction time $(p<0.05)$ (Fig. 2g). From the results above, we could see that BDNF, NGF, and NT3 were secreted continuously during the induction of hAMSCs into SCLCs and that their secretion increased with the prolongation of induction time $(p<0.05)$. 
4Fig. 2 The expression of S-100, P75, and GFAP was significantly increased after differentiation from hAMSCs into SCLCs and the expression of BDNF, NGF, and NT3 also markedly increased during differentiation. (a) The culture and morphological identification of SCLCs at day 0 (before differentiation), day 7, day 14, and day 21 after differentiation induction (scale bar $100 \mu \mathrm{m}$ ). (b-d) Immunofluorescence detection expression of S-100 (b), P75 (c), and GFAP (d) in undifferentiated hAMSCs or SCLCs after differentiation for 21 days. The undifferentiated hAMSC group was used as the negative control. The fluoroscopic time was set to $500 \mathrm{~ms}$. S-100, P75, and GFAP were located in the cytoplasm of SCLCs (scale bar $100 \mu \mathrm{m}$ ). (e) Detection of S-100, P75, and GFAP expression in undifferentiated hAMSCs or SCLCs after differentiation for 21 days by qPCR. (f) Detection of S-100, $\mathrm{P} 75$, and GFAP expression in undifferentiated hAMSCs or SCLCs after differentiation for 21 days by western blot. (g) Detection of BDNF, NGF, and NT3 level in supernatant during induction by ELISA at day 0, day 3 , day 14 , and day 21 . Data represent mean \pm S.D. $* P<0.05$ MiRNA sequencing and functional analysis
of differentially expressed miRNAs during the differentiation from hAMSCs into SCLCs

Based on cutoff criteria of absolute value of $\log F C \geq 1$ and $p$ value $<0.05,443$ differentially expressed miRNAs were obtained in the miR-seq experiment. The top 20 (top 10 up-regulated and top 10 down-regulated) miRNAs with the highest $\log _{2} \mathrm{FCl}$ values were selected as SCLCs-related miRNAs (Fig. 3a). Through the prediction of miRNA-RNA databases (DIANA TOOLS, miRTarBase and Targetscan), 2460 common target genes of the top 20 SCLCs-related miRNAs were found (Fig. 3b). According to the prediction of selected target genes of the top 20 miRNAs, Kyoto

b

16.00

14.00

12.00

10.00

8.00

6.00

4.00

2.00 0.00
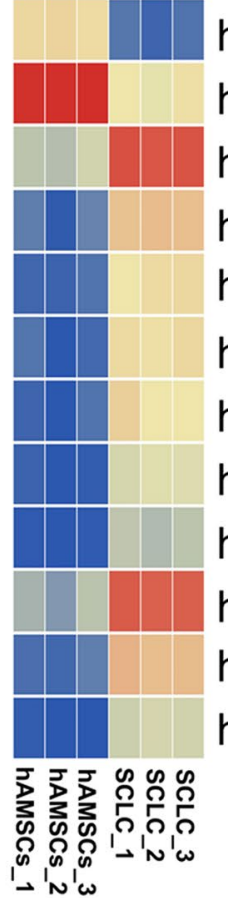

hsa-miR-146a-3p

hsa-miR-31-5p

hsa-miR-493-5p

hsa-miR-10a-3p

hsa-miR-127-3p

hsa-miR-493-3p

hsa-miR-432-5p

hsa-miR-136-3p

hsa-miR-146a-5p

hsa-miR-218-5p

hsa-miR-20b-5p

hsa-miR-375

hsa-miR-135a-5p

hsa-miR-338-3p

hsa-miR-218-2-3p

hsa-miR-488-3p

hsa-miR-9-5p

hsa-miR-592

hsa-miR-1269b
Fig. 3 MiRNA sequencing and functional analysis of differentially expressed miRNAs during the differentiation from hAMSCs into SCLCs. a Heatmap of TOP 20 differentially expressed miRNAs before and after SCLC differentiation for 14 days by miRNA-seq analysis. b Overlapped predicted targets of miR-146a-3p of miRNA databases 


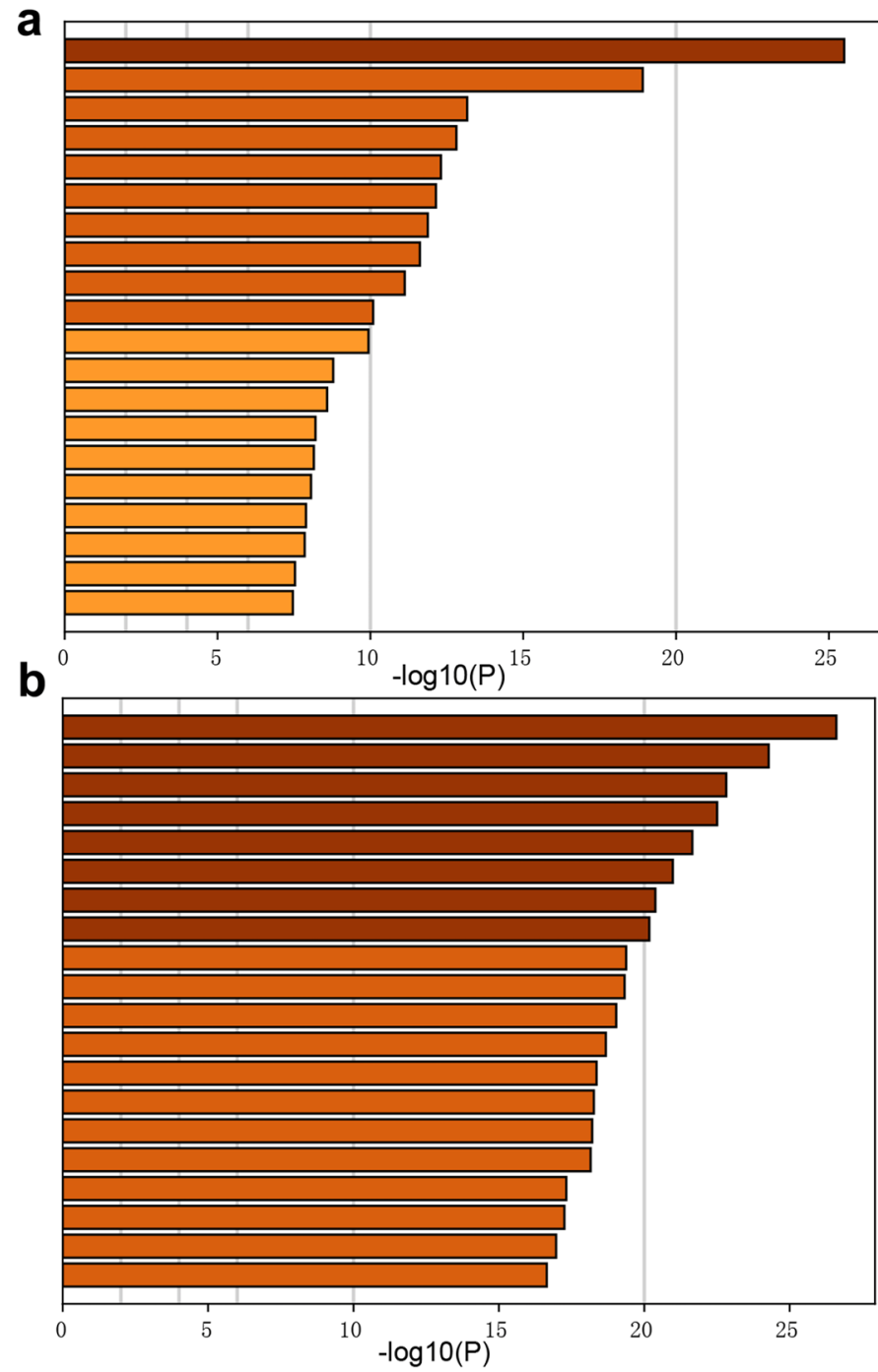

hsa05200: Pathways in cancer

hsa05205: Proteoglycans in cancer

hsa04520: Adherens junction

hsa05161: Hepatitis B

hsa04144: Endocytosis

hsa05166: HTLV-I infection

hsa05203: Viral carcinogenesis

hsa04390: Hippo signaling pathway

hsa04360: Axon guidance

hsa05202: Transcriptional misregulation in cancer

hsa04152: AMPK signaling pathway

hsa04120: Ubiquitin mediated proteolysis

hsa04550: Signaling pathways regulating pluripotency of stem cells hsa04933: AGE-RAGE signaling pathway in diabetic complications

hsa01521: EGFR tyrosine kinase inhibitor resistance

hsa05034: Alcoholism

hsa04530: Tight junction

hsa04110: Cell cycle

hsa04919: Thyroid hormone signaling pathway

hsa04140: Autophagy - animal

GO:0006325: chromatin organization

GO:0000904: cell morphogenesis involved in differentiation GO:0060322: head development

GO:0080135: regulation of cellular response to stress GO:0031329: regulation of cellular catabolic process GO:0044403: symbiont process

GO:0070848: response to growth factor

GO:0048729: tissue morphogenesis

GO:0009611: response to wounding

GO:0030029: actin filament-based process

GO:0016567: protein ubiquitination

GO:0010942: positive regulation of cell death

GO:0016055: Wnt signaling pathway

GO:0051347: positive regulation of transferase activity

GO:0030099: myeloid cell differentiation

GO:0034330: cell junction organization

GO:0097190: apoptotic signaling pathway

GO:0070482: response to oxygen levels

GO:0007169: transmembrane receptor protein tyrosine kinase signaling GO:0048732: gland development

\section{Down-regulation}

C

$$
\text { C }
$$
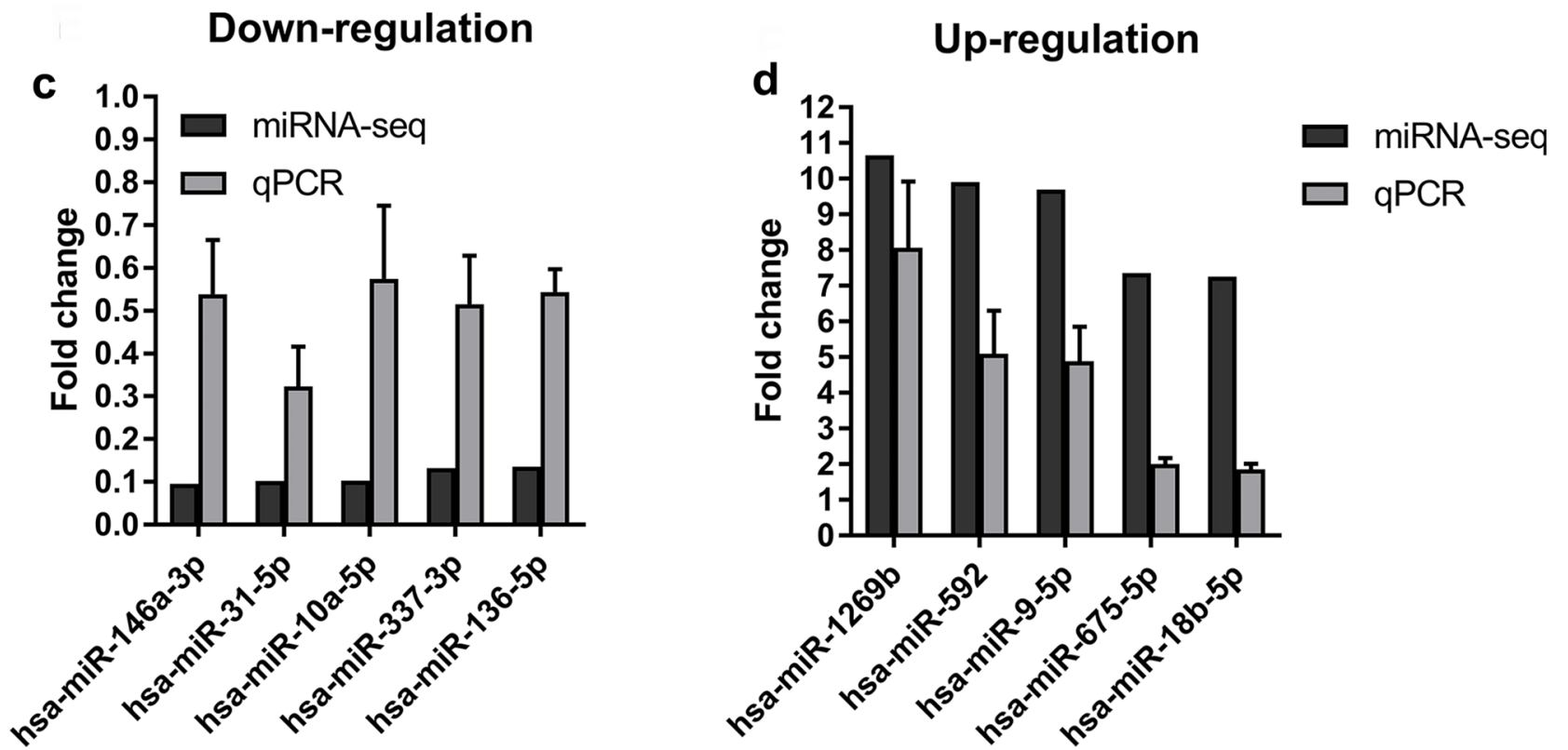

d

\section{Up-regulation}

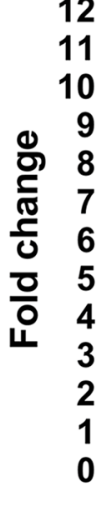

miRNA-seq

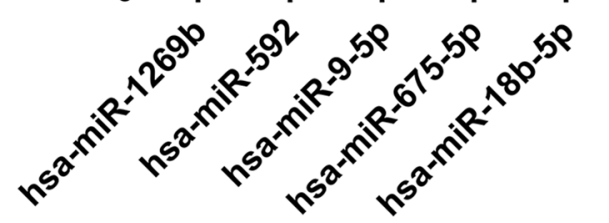


4Fig. 4. a KEGG pathway enrichment analysis of differentially expressed microRNAs before and after SCLC differentiation at day 14. b Gene ontology analysis of differentially expressed microRNAs before and after SCLC differentiation at day 14. c, d Verification of top 10 differential expressed miR-seq results by qPCR the fold changes shows the fold change of selected miRNAs between the hAMSCs and SCLC differentiation for 14 days. Data represent mean \pm S.D. $* P<0.05$

Encyclopedia of Genes and Genomes (KEGG) and Gene Ontology (GO) enrichment analysis were performed by Metascape. Top 20 GO biological process terms and KEGG pathways with significant changes before and after induction are shown in Fig. $4 \mathrm{a}$ and b. KEGG and GO enrichment analysis demonstrated that the top 20 miRNAs implicated in cancer development, cell junction, and more importantly, cell differentiation related pathway, such as cell morphogenesis involved in differentiation and Wnt signalling pathway, Hippo signaling pathway signaling, and pathway regulating pluripotency of stem cells. Next, the top 10 miRNAs (top 5 up-regulated and top 5 down-regulated) were selected to verify the expression before and after induction by qPCR. The expression trend of the selected miRNAs was consistent with that of the sequencing results (Fig. $4 \mathrm{c}$ and d), indicating that the sequencing results of miRNAs were reliable and could be used for further experiments.

\section{ERBB2 is the direct downstream target of mir-146a-3p, which regulated the expression of S-100, P75, and GFAP in cells and the secretion of BDNF, NGF, and NT3}

Combining the literature with the above analysis results, we selected the miRNA that showed the largest downregulation during the differentiation, miR-146a-3p as the research target. MiRNA target prediction study showed that an important differentiation regulator ERBB2 may be the target of miR$146 \mathrm{~A}-3 \mathrm{p}$ by binding to the $3^{\prime}$-UTR of ERBB2. Then, we hypothesized that miR-146a-3p regulated the differentiation from hAMSCs into SCLCs via targeting ERBB2. In order to confirm the hypothesis, cells were collected at days 0 , 7,14 , and 21 during the differentiation of hAMSCs into SCLCs. The expression of miR-146a-3p and EBBB2 were measured by qPCR. The result showed that the levels of miR$146 a-3 p$ decreased and those of ERBB2 increased with the increase of the induction time and that there was a negative correlation between them (Fig. 5a). The result suggested that during differentiation, miR-146a-3p might play negatively regulated role during differentiation. The downregulation of miR-146a-3p might release its restoration on downstream target ERBB2, and cell differentiation was promoted by the upregulation of ERBB2.
MiR-146a-3p mimics and inhibitors were transfected into hAMSCs before Schwann cell differentiation for 7 days to further study the function of miR-146a-3p. The immunofluorescence analyses suggested that after inhibiting miR-146a-3p, the expression of S-100, P75, and GFAP were significantly higher than those in the control groups (Fig. 5b-d). The results from qPCR and western bolt also confirmed the finding (Fig. $4 \mathrm{e}$ and $\mathrm{f}$ ). The results above showed that transfection of miR-146a-3p inhibitors could induce the up-regulation of neurocyte-specific proteins and promote differentiation from hAMSCs into SCLCs.

The levels of BDNF, NGF, and NT3 in the supernatant of SCLCs after miR-146a-3p over-expression or interference for 7 days were measured by ELISA. The results showed that the secretion of neurotrophic factors increased after overexpressing miR-146a-3p and decreased after interfering with miR-146a-3p (Fig. 5g). This experiment suggested that miR$146 \mathrm{a}-3 \mathrm{p}$ might regulate the secretion of neurotrophic factors.

Furthermore, to verify the functions of ERBB2, siRNAs of ERBB2 were transfected into hAMSCs before Schwann-like cell differentiation for 7 days. The qPCR, western blot, and immunofluorescence analysis suggested that the expression of Schwann cell markers S-100, P75, and GFAP were significantly down-regulated in si-ERBB2 group than those in the control groups (Fig. 6a, b, d-f). ELISA assays also showed that interfering the expression of ERBB2 reduced the concentration of BDNF, NGF, and NT3 in the supernatant of SCLCs (Fig. 6c). The experiment showed that ERBB2 functioned a positive role in Schwann-like cell differentiation from hAMSCs.

It can be inferred from the above results that miR-146a-3p may inhibited the differentiation of hAMSCs into SCLCs by downregulating the expression of ERBB2.

\section{Discussion}

Nowadays, autologous nerve transplantation is still the "golden standard" for repairing peripheral nerve defects, but there are several drawbacks such as limited sources of donors, residual donor site sensory dysfunction, and increased donor site trauma. For peripheral nerve growth, various drugs or nutrition factors are used to promote peripheral nerve repair and regeneration, but their clinical effects are limited (Menorca et al. 2013). Schwann cells, which are unique glial cells of the peripheral nervous system, can promote the repair and regeneration of peripheral nerve tissue. After a peripheral nerve injury, SCs phagocytize deformed axons and myelin sheath debris and rapidly proliferate to form Bungner bands, secrete neurotrophic factors, extracellular matrix, and adhesion molecules, guide axon growth direction, maintain neuronal vitality, and promote the peripheral reintroduction 


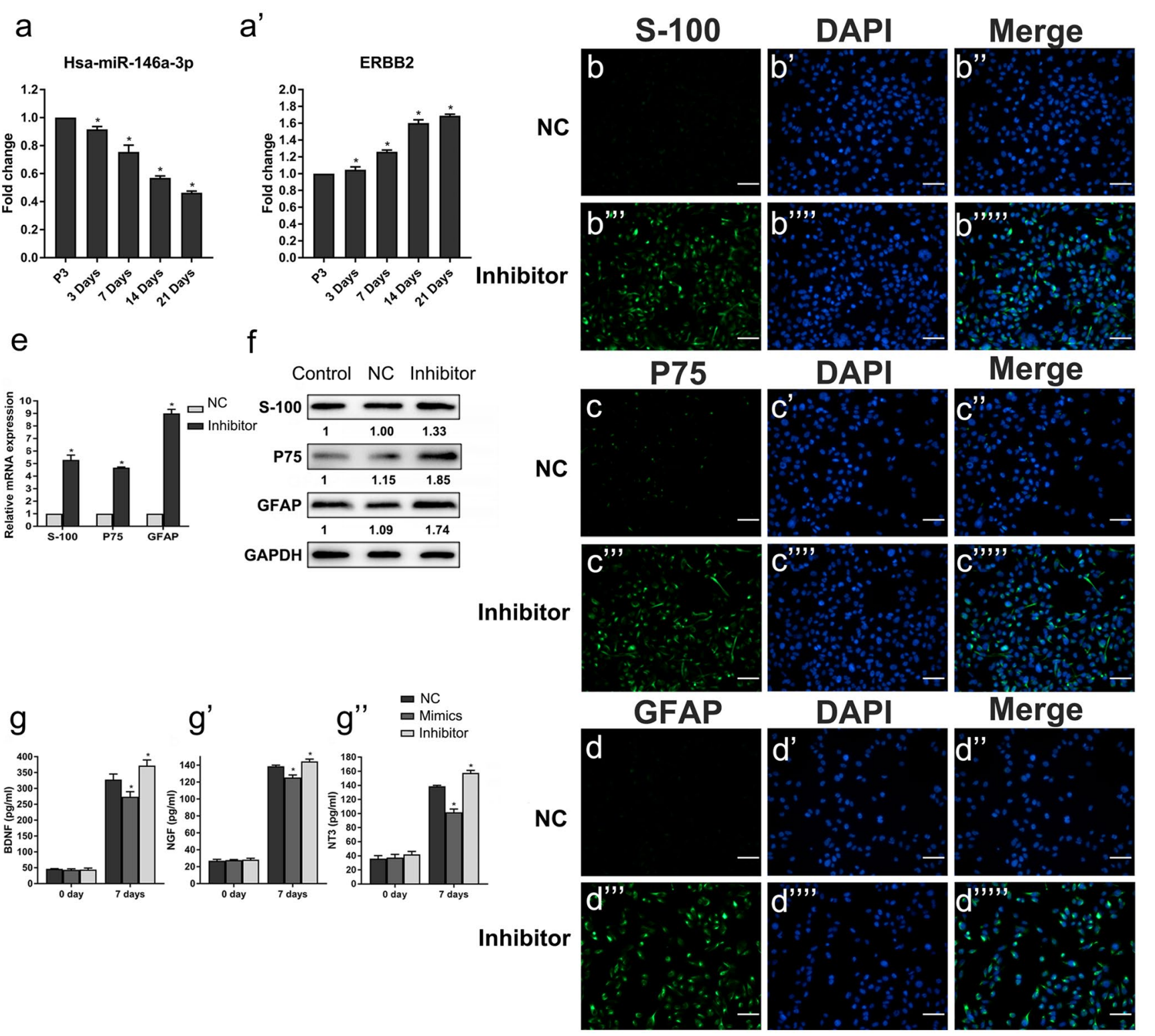

Fig. 5 ERBB2 is the direct downstream target of mir-146a-3p, which regulated the expression of S-100, P75, and GFAP and the secretion of BDNF, NGF, and NT3 in the cells. SCLCs were divided into three groups, SCLCs without transfection (control group), SCLCs transfected with negative control sequence (NC group), SCLCs transfected with inhibitor of miR-146a-3p (inhibitor group) SCLCs transfected with miR-146a-3p mimics (mimics group). (a) Changes of the expressions of miR-146a-3p and ERBB2 during Schwann-like induction. (b-d) Immunofluorescence detection of the effect of miR-146a-3p on the expression of S-100 (b), P75 (c), and GFAP (d) of SCLCs after

of the injured area (Stoll et al. 1989). However, a key problem has been encountered regarding the acquisition of highly purified SCs (Stefanescu et al. 2012).

Herein, we aimed to derive fate-committed SCLCs from hAMSCs such that sufficient numbers could be tapped on demand for transplantation to improve the prospects of the differentiation at day 14. SCLCs transfected with negative control sequence was used as the negative control. The fluoroscopic time was set to $500 \mathrm{~ms}$. S-100, P75, and GFAP were located in the cytoplasm of SCLCs (scale bar $100 \mu \mathrm{m}$ ). (e) Detection of the effect of miR$146 \mathrm{a}-3 \mathrm{p}$ on the expression of S-100, P75, and GFAP in NC group and inhibitor group at day 14 by qPCR. (f) Detection of the effect of miR$146 \mathrm{a}-3 \mathrm{p}$ on the expression of S-100, P75, and GFAP in NC group and inhibitor group at day 14 by western blot. (g) ELISA detection of the effect of miR-146a-3p on the expression of BDNF, NGF, and NT3 in three groups. Data represent mean \pm S.D. $* P<0.05$

post-traumatic re-growth and re-myelination of axons and recovery of function. With the use of stage-specific culture conditions, we achieved the goal of generating a high number of iSCs. The SCLCs displayed Schwann-like molecular phenotypic characteristics, such as the neurocyte-specific proteins S-100, P75, and GFAP and the neurotrophic factors 

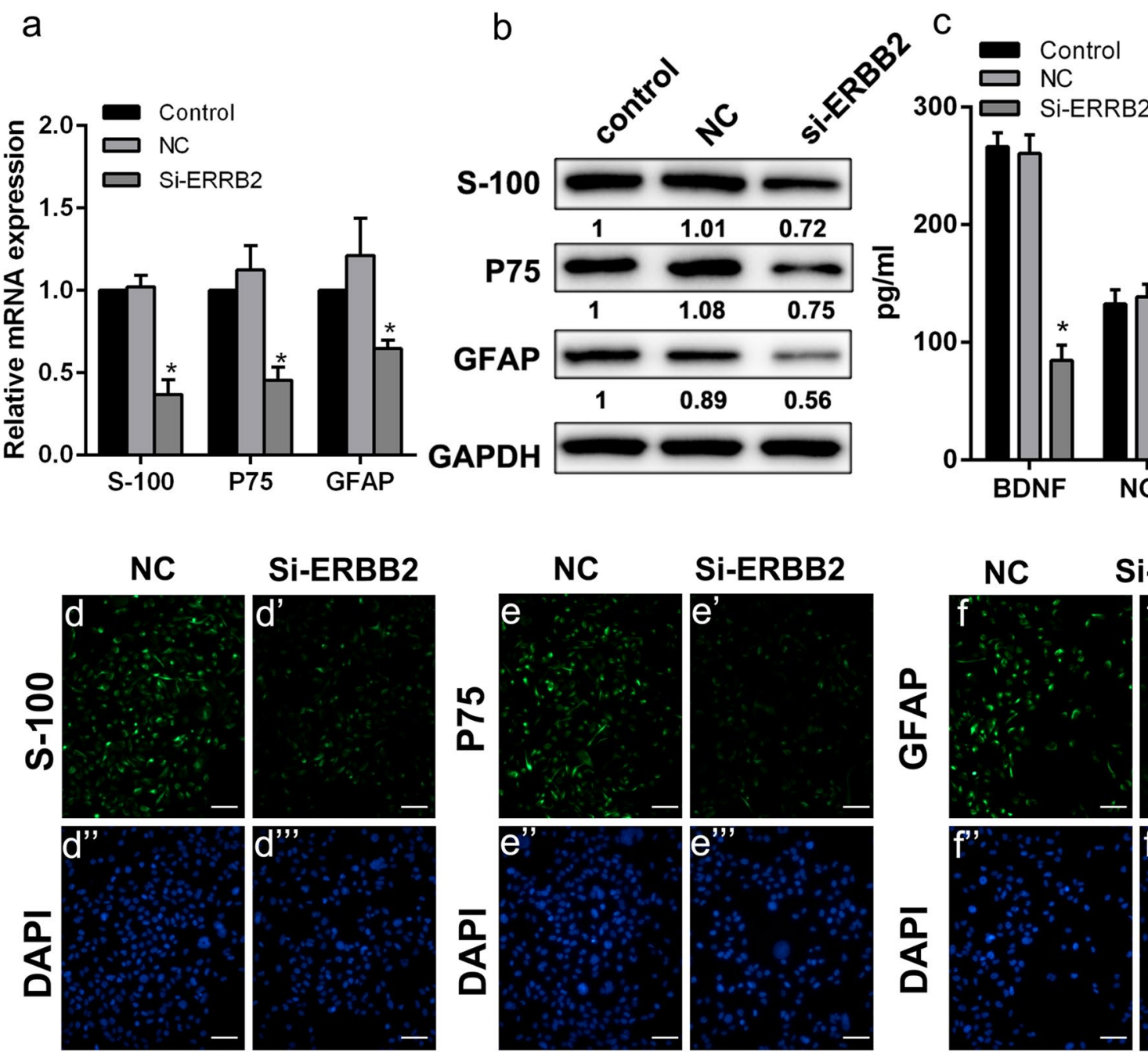

Si-ERBB2
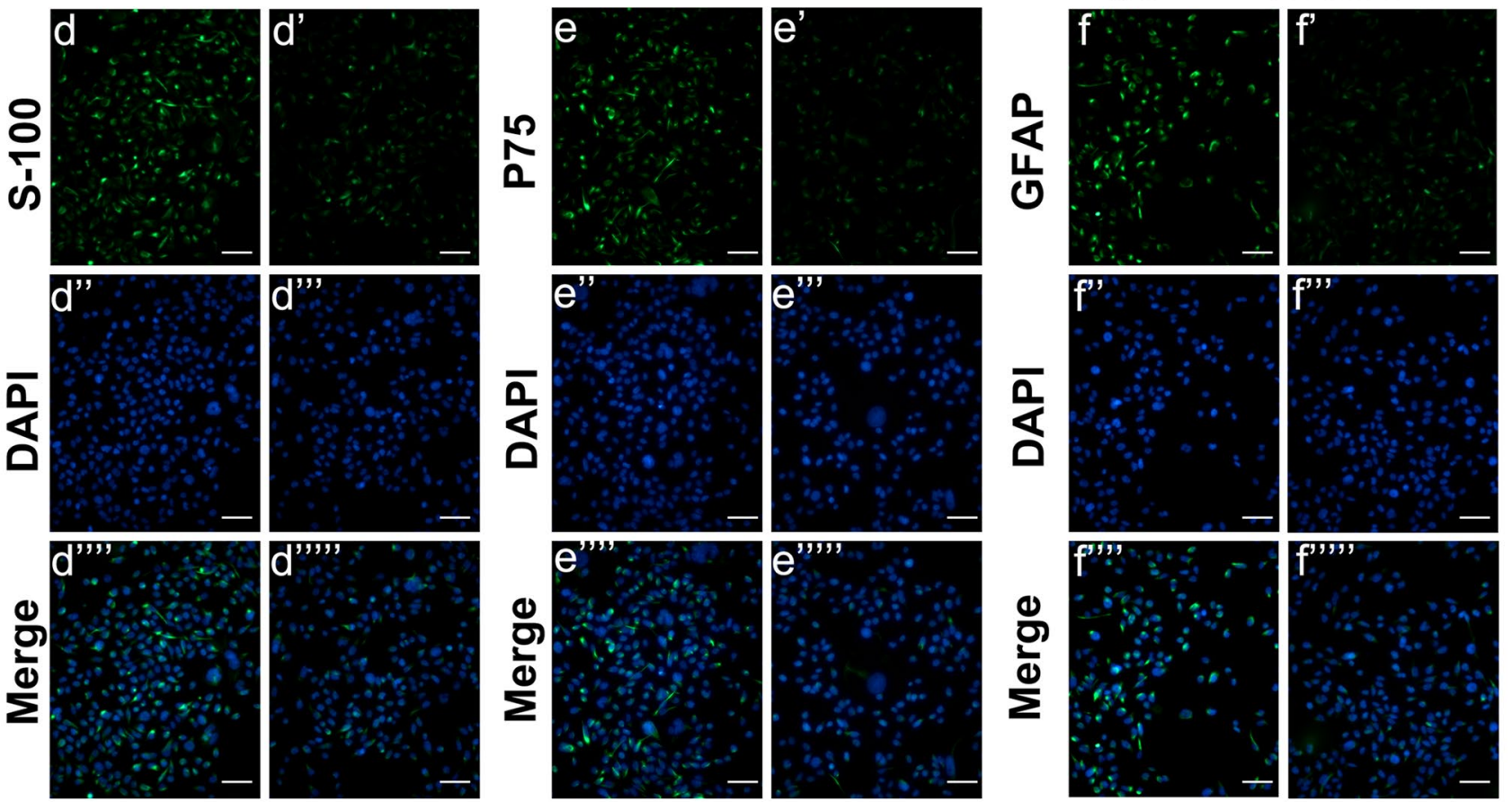

Fig. 6 ERBB2 regulated the expression of S-100, P75, and GFAP and the secretion of BDNF, NGF, and NT3 in the SCLCs cells. SCLCs cells were divided into three groups: SCLCs without transfection (control group), SCLCs transfected with negative control sequence (NC group), SCLCs transfected with siRNA of ERBB2 (Si-ERBB2 group). (a) Detection of the effect of ERBB2 on the expression of S-100, P75, and GFAP by qPCR in three groups at day 7. (b) Detection of the effect of ERBB2 on the expression of S-100, P75 and

BDNF, NGF, and NT3, as we expected. S-100, P75, and GFAP are neurocyte-specific markers that play an important role in peripheral nerve injury repair. Their expression indicates the formation of Schwann cells (Rutten et al. 2012). BDNF, NGF, and NT3 are members of the Schwann cell-derived neurotrophic factor family, which is closely related to the
GFAP by western blot at day 7 in three groups. (c) ELISA detection of the effect of ERBB2 on the expression of BDNF, NGF, and NT3 in three groups. (d-f) Immunofluorescence detection of the effect of ERBB2 on the expression of S-100, P75, and GFAP SCLCs transfected with negative control sequence was used as the negative control, the fluoroscopic time was set to $500 \mathrm{~ms}$. S-100, P75, and GFAP were located in the cytoplasm of SCLCs (scale bar $100 \mu \mathrm{m}$ ). Data represent mean \pm S.D. $* P<0.05$

regeneration and differentiation of neurons. After peripheral nerve injury, NGF, BDNF, and NT3 are secreted and bind their high-affinity receptors trkA, trkB, and trkC and, then, activate the tyrosine kinase signaling system (Boyd and Gordon 2003). During repair, neurotrophic factors can avoid neuronal retrograde death after peripheral nerve injury, guide 
and promote axon regeneration, and promote myelin sheath formation. Our results indicated that SCLCs have the potential to function as mature human Schwann cells in the repair of peripheral nerve injury, which was consistent with the previous reports (Gon et al. 2018; Sanluis-Verdes et al. 2017).

Results from this study also showed that miR-146a-3p expression was clearly decreased at day 14 post-induction, approximately 0.4 -fold less than prior to induction, further increased thereafter, and tended to stabilize at day 30 . Together with a previous finding that miR-146a expression was down-regulated after acute intracerebral hemorrhage, we hypothesized that miR-146a plays a regulatory role in the process of hAMSC differentiation into Schwann cells (Zh et al. 2015).

The miR-146a gene is located in 5q34 and plays roles in diverse fields, including tumorigenesis, respiratory diseases pathogenesis, and immune reaction. MiRNA-146a was previously reported to be involved in cancer development and autoimmune disease (Bogunia-Kubik et al. 2016; Kotlarek et al. 2018; Marega et al. 2016). Recently, Hsa-miR-146a-3p was found be to a regulator of macrophage differentiation and M1/M2 polarized activation processes (Essandoh et al. 2016). Yi reported that hsa-miR-146a-3p negatively regulated the adipogenesis from human bone mesenchymal stem cells via targeting PLIN4 (Yi et al. 2019).

Results from this study showed that after interference with miR-146a-3p by using miR-146a-3p-inhibitor, induced SCLCs had an increased expression of Schwann cellsrelated neurotrophic factors while, after over-expression of miR-146a-3p, the differentiation capacity was significantly attenuated. It is noteworthy, this indicated that miR$146 a-3 p$ might function as a negative regulator not only on adiposegenes from hBMSCs as well as the not the differentiation of hAMSCs into SCLCs. Therefore, the role of miR-146a-3p in other MSC differentiation is worth further exploring. To investigate the mechanism by which miR$146 \mathrm{a}-3 \mathrm{p}$ regulated the differentiation of neural crest stem cells, we sought to find miR-146a-3p targets via databases. Results showed that a 7-mer-long sequence at the 3'UTR region of the ERBB2 mRNA was matched to miR-146-3p. ERRB2 encodes a member of the epidermal growth factor (EGF) receptor family of receptor tyrosine kinases. ERRB2 mainly exerted its regulatory function of specific receptor of neuregulin-1 (NRG1) by forming dimers with other EGF receptors. NRG1-ERRB2 signal pathways partake in the regulation of growth and development of glial cells, enteric neurons, and cardiomyocytes (Barrenschee et al. 2015; Grazette et al. 2004; Schmid et al. 2003).

In the peripheral nervous system, the number of SCs should correspond to the number of axons. The ERBB signal is clearly active during the development of SCs. It has been confirmed that inhibition of the ERBB tyrosine kinase receptor can inhibit the proliferation of SCs and promote their apoptosis, further affecting the migration of SCs to axonal terminals and axonal transplantation after nerve injury (Suzana et al. 2006). In addition, if a mutation occurs in ERBB, SCs' transplantation to axons will be severely damaged (Lyons et al. 2005). Therefore, the ERBB signal is very important for the normal function of SCs. Thus, this study selected ERBB2 as a potential target of miR-146a-3p and investigated whether miR-146a-3p regulated the differentiation of amniotic mesenchymal stem cells by influencing the expression of ERBB2. We found that the expression of miR-146a-3p decreased and the expression of ERBB2 increased during the differentiation period. This suggested that ERBB2 was likely to be a down-stream target gene via which miR-146a-3p functions. Based on this finding, targeting miRNA-146a-3p expression during the differentiation of hAMSCs to SCLCs be a practical method to promote the production of SCLCs. However, further vivo experiments need to explore the efficacy and safety of SCLCs produced by this method to peripheral nerve injury.

\section{Conclusion}

Taken together, this study demonstrated that human amniotic membrane stem cells could be differentiated into functional Schwann cell-like cells. By miRNA-seq, we identified that miRNAs were significantly associated with Schwann celllike differentiation of hAMSCs. During the differential procedure, miR-146a-3p might play negative role through the down-regulating of the ERBB2 protein expression. The results might provide a new insight for batch acquisition of purified Schwann cells and further development into an autologous cell source for implantation as a safe therapy for nerve injuries or peripheral neuropathies.

Author contributions Zairong Wei and Wei Chen investigated the study, responsible for data curation, carried out the formal analysis, visualized the data, and wrote the original draft of the manuscript. Wei Chen, Linlin Ji, Zairong Wei, Chenglan Yang, and Shusen Chang investigated the study and carried out the methodology and data validation. Wei Chen, Yucheng Zhang, Kaiyu Nie, Lingli Jiang, and Yurong Deng investigated the study and carried out the formal analysis. Zairong Wei and Wei Chen conceptualized the study, involved in funding acquisition, and carried out the project administration. Wei Chen, Linlin Ji, and Zairong Wei were responsible for the resources, supervised the study, and wrote, reviewed, and edited the manuscript. All authors made the substantial contributions to the manuscript and approved its final version.

Funding This study was supported by grants from the National Natural Science Foundation of China (81760347), Science and Technology Support Plan Fund of Guizhou Province ([2017]2877), National Natural Science Foundation of China (81560315), Science and Technology Department Fund of Guizhou Province (2019 (4441)), and Adiposederived Stem Cell Matrigel Foundation and Clinical Application Research Innovation Talent Team of Zunyi City ([2018]9). 


\section{Compliance with ethical standards}

Conflict of interest The authors declare that they have no conflict of interest.

Ethical approval This article does not contain any studies with human participants or animals performed by any of the authors.

Open Access This article is licensed under a Creative Commons Attribution 4.0 International License, which permits use, sharing, adaptation, distribution and reproduction in any medium or format, as long as you give appropriate credit to the original author(s) and the source, provide a link to the Creative Commons licence, and indicate if changes were made. The images or other third party material in this article are included in the article's Creative Commons licence, unless indicated otherwise in a credit line to the material. If material is not included in the article's Creative Commons licence and your intended use is not permitted by statutory regulation or exceeds the permitted use, you will need to obtain permission directly from the copyright holder. To view a copy of this licence, visit http://creativecommons.org/licenses/by/4.0/.

\section{References}

Armaiz FA, Wang H (2017) The use and delivery of stem cells in nerve regeneration: preclinical evidence and regulatory considerations. Ann Plast Surg 80:448-456

Avendaño-Félix M, Fuentes-Mera L, Ramos-Payan R, Aguilar-Medina M, Pérez-Silos V, Moncada-Saucedo N, Marchat LA, GonzálezBarrios JA, Ruiz-García E, Astudillo-de la Vega H, Cruz-Colin JL, López-Camarillo C (2019) A novel OsteomiRs expression signature for osteoblast differentiation of human amniotic membrane-derived mesenchymal stem cells. Biomed Res Int 2019:8987268-8987268

Barrenschee M, Lange C, Cossais F, Egberts JH, Becker T, Wedel $\mathrm{T}$, Bottner M (2015) Expression and function of Neuregulin 1 and its signaling system ERBB2/3 in the enteric nervous system. Frontiers in cellular neuroscience 9:360

Bogunia-Kubik K, Wysoczańska B, Piątek D, Iwaszko M, Ciechomska M, Świerkot J (2016) Significance of polymorphism and expression of miR-146a and NFkB1 genetic variants in patients with rheumatoid arthritis. Archivum immunologiae et therapiae experimentalis 64:131-136

Boyd JG, Gordon T (2003) Neurotrophic factors and their receptors in axonal regeneration and functional recovery after peripheral nerve injury. Mol Neurobiol 27:277-324

Carbone A, Paracchini V, Castellani S, Di Gioia S, Seia M, Colombo C, Conese M (2014) Human amnion-derived cells: prospects for the treatment of lung diseases. Curr Stem Cell Res Ther 9:297-305

Carr MJ, Johnston AP (2017) Schwann cells as drivers of tissue repair and regeneration. Curr Opin Neurobiol 47:52-57

Diaz-Prado S, Muinos-Lopez E, Hermida-Gomez T, Cicione C, Rendal-Vazquez ME, Fuentes-Boquete I, de Toro FJ, Blanco FJ (2011) Human amniotic membrane as an alternative source of stem cells for regenerative medicine. Differentiation 81 : $162-171$

Dominici M, Le Blanc K, Mueller I, Slaper-Cortenbach I, Marini F, Krause D, Deans R, Keating A, Prockop D, Horwitz E (2006) Minimal criteria for defining multipotent mesenchymal stromal cells. The International Society for Cellular Therapy position statement. Cytotherapy 8:315-317

Essandoh K, Li Y, Huo J, Fan GC (2016) MiRNA-mediated macrophage polarization and its potential role in the regulation of inflammatory response. Shock (Augusta, Ga) 46:122-131
Glemžaitė M, Navakauskienė R (2016) Osteogenic differentiation of human amniotic fluid mesenchymal stem cells is determined by epigenetic changes. Stem cells international 2016:6465307-6465307

Gong F, Wei Z, Jin W, Li H, Deng C, Wu B, Nie K (2018) [The role of Schwann cells-like cells derived from human amniotic membrane mesenchymal stem cells transplantation in flap nerves regeneration]. Zhongguo xiu fu chong jian wai ke za zhi $=$ Zhongguo xiufu chongjian waike zazhi $=$ Chinese journal of reparative and reconstructive surgery 32:80-90

Grazette LP, Boecker W, Matsui T, Semigran M, Force TL, Hajjar RJ, Rosenzweig A (2004) Inhibition of ErbB2 causes mitochondrial dysfunction in cardiomyocytes : Implications for herceptin-induced cardiomyopathy. J Am Coll Cardiol 44:2231-2238

Jiang L, Jones S, Jia X (2017) Stem cell transplantation for peripheral nerve regeneration: current options and opportunities. Int J Mol Sci 18:94

Jiang TM, Yang ZJ, Kong CZ, Zhang HT (2010) Schwann-like cells can be induction from human nestin-positive amniotic fluid mesenchymal stem cells. Vitro Cell Dev Biol Anim 46:793-800

Koike C, Zhou K, Takeda Y, Fathy M, Okabe M, Yoshida T, Nakamura Y, Kato Y, Nikaido T (2014) Characterization of amniotic stem cells. Cellular reprogramming 16:298-305

Kotlarek M, Kubiak A, Czetwertyńska M, Świerniak M, Gierlikowski W, Kolanowska M, Bakuła-Zalewska E, Jhiang SM, Jażdżewski K, Wójcicka A (2018) The rs2910164 genetic variant of miR$146 a-3 p$ is associated with increased overall mortality in patients with follicular variant papillary thyroid carcinoma. Int J Mol Sci 19:

Lyons DA, Hans-Martin P, Voas MG, Woods IG, Brianne D, Rebecca N, Naomi A, Jennifer J, Talbot WS (2005) erbb3 and erbb2 are essential for Schwann cell migration and myelination in zebrafish. Curr Biol 15:513-524

Marega LF, Teocchi MA, Dos Santos Vilela MM (2016) Differential regulation of miR-146a/FAS and miR-21/FASLG axes in autoimmune lymphoproliferative syndrome due to FAS mutation (ALPS-FAS). Clin Exp Immunol 185:148-153

Martin EC, Qureshi AT, Dasa V, Freitas MA, Gimble JM, Davis TA (2016) MicroRNA regulation of stem cell differentiation and diseases of the bone and adipose tissue: Perspectives on miRNA biogenesis and cellular transcriptome. Biochimie 124:98-111

Menorca RM, Fussell TS, Elfar JC (2013) Nerve physiology: mechanisms of injury and recovery. Hand Clin 29:317-330

Mok GF, Lozano-Velasco E, Munsterberg A (2017) microRNAs in skeletal muscle development. Semin Cell Dev Biol 72:67-76

Nardelli C, Granata I, Iaffaldano L, D'Argenio V, Del Monaco V, Maruotti GM, Omodei D, Del Vecchio L, Martinelli P, Salvatore F, Guarracino MR, Sacchetti L, Pastore L (2017) miR-138/miR222 overexpression characterizes the miRNome of amniotic mesenchymal stem cells in obesity. Stem cells and development 26:4-14

Romani R, Pirisinu I, Calvitti M, Pallotta MT, Gargaro M, Bistoni G, Vacca C, Di Michele A, Orabona C, Rosati J, Pirro M, Giovagnoli S, Matino D, Prontera P, Rosi G, Grohmann U, Talesa VN, Donti E, Puccetti P, Fallarino F (2015) Stem cells from human amniotic fluid exert immunoregulatory function via secreted indoleamine 2,3-dioxygenase1. J Cell Mol Med 19:1593-1605

Rutten MJ, Janes MA, Chang IR, Gregory CR, Gregory KW (2012) Development of a functional schwann cell phenotype from autologous porcine bone marrow mononuclear cells for nerve repair. Stem cells international 2012:738484

Sanluis-Verdes A, Sanluis-Verdes N, Manso-Revilla MJ, CastroCastro AM, Pombo-Otero J, Fraga-Mariño M, Sanchez-Ibañez J, Doménech N, Rendal-Vázquez ME (2017) Tissue engineering for neurodegenerative diseases using human amniotic membrane and umbilical cord. Cell Tissue Banking 18:1-15 
Schmid RS, Barbara MG, Berechid BE, Becky B, Mark M, Nenad S, Anton ES (2003) Neuregulin 1-erbB2 signaling is required for the establishment of radial glia and their transformation into astrocytes in cerebral cortex. Proc Natl Acad Sci U S A 100:4251-4256

Stefanescu O, Enescu DM, LascaR L (2012) Schwann cell cultures: recent advances and novel approaches to the reconstruction of peripheral nerve defects. Rom J Morphol Embryol 53:467-471

Stoll G, Griffin JW, Li CY, Trapp BD (1989) Wallerian degeneration in the peripheral nervous system: participation of both Schwann cells and macrophages in myelin degradation. J Neurocytol 18:671

Suzana A, Scherer SS, Erich S, Dino L, Garratt AN, Carmen B, Ueli $S$ (2006) ErbB2 signaling in Schwann cells is mostly dispensable for maintenance of myelinated peripheral nerves and proliferation of adult Schwann cells after injury. Journal of Neuroscience the Official Journal of the Society for Neuroscience 26:2124-2131

Trohatou O, Zagoura D, Bitsika V, Pappa KI, Antsaklis A, Anagnou NP, Roubelakis MG (2014) Sox2 suppression by miR-21 governs human mesenchymal stem cell properties. Stem cells translational medicine 3:54-68

Trohatou O, Zagoura D, Orfanos NK, Pappa KI, Marinos E, Anagnou NP, Roubelakis MG (2017) miR-26a mediates adipogenesis of amniotic fluid mesenchymal stem/stromal cells via PTEN, Cyclin E1, and CDK6. Stem cells and development 26:482-494

Wang AT, Zhang QF, Wang NX, Yu CY, Liu RM, Luo Y, Zhao YJ, Xiao JH (2020) Cocktail of hyaluronic acid and human amniotic mesenchymal cells effectively repairs cartilage injuries in sodium iodoacetate-induced osteoarthritis rats. Front Bioeng Biotechnol 8:87

Yi X, Liu J, Wu P, Gong Y, Xu X, Li W (2019) The key microRNA on lipid droplet formation during adipogenesis from human mesenchymal stem cells. J Cell Physiol 235:328-338

Zheng YB, Gao ZL, Xie C, Zhu HP, Peng L, Chen JH, Chong YT (2008) Characterization and hepatogenic differentiation of mesenchymal stem cells from human amniotic fluid and human bone marrow: a comparative study. Cell Biol Int 32:1439-1448

Zhu Y, Wang JL, He ZY, Jin F, Tang L (2015) Association of altered serum MicroRNAs with perihematomal edema after acute intracerebral hemorrhage. PLoS One 10:e0133783

Publisher's Note Springer Nature remains neutral with regard to jurisdictional claims in published maps and institutional affiliations. 